\title{
A Behavioral Approach to LPV Systems
}

\author{
Roland Tóth, Jan C. Willems, Peter S. C. Heuberger, and Paul M. J. Van den Hof
}

\begin{abstract}
Linear Parameter-Varying (LPV) systems are usually described in either state-space or input-output form. When analyzing system equivalence between different models it appears that time-shifted versions of the scheduling signal (dynamic dependence) need to be taken into account. In order to construct a parametrization-free description of LPV systems a behavioral approach is introduced that serves as a solid basis for specifying system theoretic properties. LPV systems are defined as the collection of valid trajectories of system variables (like inputs and outputs) and scheduling variables. Kernel, inputoutput, and state-space representations are introduced as well as appropriate equivalence transformations between these models.
\end{abstract}

Index Terms-LPV, behavioral approach, dynamic dependency, equivalence.

\section{INTRODUCTION}

Many physical/chemical processes exhibit parameter variations due to non-stationary or nonlinear behavior, or dependence on external variables. For such processes, the theory of Linear Parameter-Varying (LPV) systems offers an attractive modeling framework [1]. This class of systems is particularly suited to deal with systems that operate in varying operating regimes. LPV systems can be seen as an extension of the class of Linear Time-Invariant (LTI) systems. In LPV systems, the signal relations are considered to be linear, but the model parameters are assumed to be functions of a time-varying signal, the so-called scheduling variable $p$. As a result of this parameter variation, the LPV system class can describe both time-varying and nonlinear phenomena. Practical use of this framework is stimulated by the fact that LPV control design is well worked out, extending results of optimal and robust LTI control theory to nonlinear, time-varying plants [1], [2], [3].

In a discrete-time setting, LPV systems are commonly described in a state-space (SS) form:

$$
\begin{aligned}
q x & =A(p) x+B(p) u \\
y & =C(p) x+D(p) u,
\end{aligned}
$$

where $u$ is the input, $y$ is the output of the system, $x$ is the state vector, $q$ is the forward time-shift operator, e.g. $q x(k)=x(k+1)$, and the system matrices $\{A, B, C, D\}$ are functions of the scheduling signal $p: \mathbb{Z} \rightarrow \mathbb{P}$, where $\mathbb{P} \subseteq \mathbb{R}^{n \mathbb{P}}$. It is assumed that $p$ is an external signal of the

This work was supported by the Dutch National Science Foundation (NWO).

R. Tóth, P.S.C. Heuberger, and P.M.J. Van den Hof are with the Delft Center for Systems and Control (DCSC), Delft University of Technology, Mekelweg 2, 2628 CD Delft, The Netherlands. r.toth@tudelft.nl

J.C. Willems is with the Department of Electrical Engineering, Katholieke Universiteit Leuven, B-3001 Leuven-Heverlee, Belgium. Jan.Willems@esat.kleuven.be system which is unknown in advance but online measurable during operation.

In the identification literature, LPV systems are also described in the form of (filter-type) input-output (IO) representations:

$$
y=\sum_{i=1}^{n_{\mathrm{a}}} a_{i}(p) q^{-i} y+\sum_{j=0}^{n_{\mathrm{b}}} b_{j}(p) q^{-j} u,
$$

where $\left\{a_{i}, b_{j}\right\}$ are functions of $p$. Note that in these descriptions the coefficients depend on the instantaneous time value of $p$. We will call such a dependence static-dependence. In analogy with the LTI system theory, it is commonly assumed that representations (1a-b) and (2) define the same class of LPV systems and that conversion between these representations follows similar rules as in the LTI case. However, it has been observed recently that this assumption is invalid if attention is restricted to static-dependency [4].

Example 1: To illustrate the problem consider the following second-order SS representation:

$$
\begin{aligned}
{\left[\begin{array}{l}
x_{1}(k+1) \\
x_{2}(k+1)
\end{array}\right] } & =\left[\begin{array}{ll}
0 & a_{2}(p(k)) \\
1 & a_{1}(p(k))
\end{array}\right]\left[\begin{array}{l}
x_{1}(k) \\
x_{2}(k)
\end{array}\right]+\left[\begin{array}{l}
b_{2}(p(k)) \\
b_{1}(p(k))
\end{array}\right] u(k), \\
y(k) & =x_{2}(k) .
\end{aligned}
$$

With simple manipulations this system can be written in an equivalent IO form:

$$
\begin{aligned}
y(k)= & a_{1}(p(k-1)) y(k-1)+a_{2}(p(k-2)) y(k-2) \\
& +b_{1}(p(k-1)) u(k-1)+b_{2}(p(k-2)) u(k-2),
\end{aligned}
$$

which is clearly not in the form defined by (2).

For obtaining equivalence between the SS and IO representations, it is necessary to allow for a dynamic mapping between $p$ and the coefficients, i.e. $\{A, B, C, D\}$ and $\left\{a_{i}, b_{j}\right\}$ should be allowed to depend on (finite many) time-shifted instances of $p(k)$, i.e. $\{\cdots, p(k-1), p(k), p(k+1), \cdots\}$ [4]. We will call such a dependence to be dynamic in the sequel.

A common ground between the several representations and concepts of LPV systems can be found by considering a behaviorial approach to the problem. In this paper the behaviorial framework, originally developed ${ }^{1}$ for LTI systems [8], is extended to LPV systems. Our aim is to establish well-defined LPV system representations as well as their interrelationships.

The paper is organized as follows: In Section II, LPV systems are defined from the behavioral point of view. In Section III, an algebraic structure of polynomials is

\footnotetext{
${ }^{1}$ In the past decades this framework has been extended to LTV ([5], [6]), and even nonlinear systems ([7], [8], [9])
} 
introduced to define parameter-varying difference equations as representations of the system behavior. This is followed by developing kernel, IO, and SS representations of LPV systems together with the basic notions of IO partitions and state-variables. In Section IV, it is explored when two kernel, IO, or SS representation are equivalent. In Section V, equivalence transformation between SS and IO representations is worked out. Finally, in Section VI, the main conclusions are summarized. In this paper, we only concentrate on discretetime systems, however analog results for the continuous-time case follow in a similar way.

\section{LPV SYSTEMS AND BEHAVIORS}

We define a parameter-varying system $\mathcal{S}$ as a quadruple

$$
\mathcal{S}=(\mathbb{T}, \mathbb{P}, \mathbb{W}, \mathfrak{B}),
$$

where $\mathbb{T} \subseteq \mathbb{R}$ is called the time axis, $\mathbb{P}$ denotes the scheduling space (i.e. $p(k) \in \mathbb{P}$ ), $\mathbb{W}$ is the signal space with dimension $n_{\mathbb{W}}$ and $\mathfrak{B} \subset(\mathbb{P} \times \mathbb{W})^{\mathbb{T}}$ is the behavior of the system $\left(\mathbb{X}^{\mathbb{T}}\right.$ stands for all maps from $\mathbb{T}$ to $\mathbb{X}$ ).

The set $\mathbb{T}$ defines the time-axis of the system, describing continuous-time $(\mathrm{CT}), \mathbb{T}=\mathbb{R}$, and discrete-time $(\mathrm{DT}), \mathbb{T}=$ $\mathbb{Z}$, systems alike, while $\mathbb{W}$ gives the range of the system signals. $\mathfrak{B}$ defines trajectories of $(\mathbb{P} \times \mathbb{W})^{\mathbb{T}}$ that are possible according to the system model. Note that there is no prior distinction between inputs and outputs in this setting.

We also introduce the so-called projected scheduling behavior

$$
\mathfrak{B}_{\mathbb{P}}=\pi_{p} \mathfrak{B}:=\left\{p \in \mathbb{P}^{\mathbb{T}} \mid \exists w \in \mathbb{W}^{\mathbb{T}} \text { s.t. }(w, p) \in \mathfrak{B}\right\},
$$

where $\pi_{p}$ denotes projection onto $\mathbb{P}^{\mathbb{T}} \cdot \mathfrak{B}_{\mathbb{P}}$ describes all possible scheduling trajectories of $\mathcal{S}$. For a given scheduling trajectory, $p \in \mathfrak{B}_{\mathbb{P}}$, we define the projected behavior as

$$
\mathfrak{B}_{p}=\left\{w \in \mathbb{W}^{\mathbb{T}} \mid(w, p) \in \mathfrak{B}\right\} .
$$

$\mathfrak{B}_{p}$ describes all possible signal trajectories compatible with $p$. With these concepts we can define discrete-time LPV systems as follows:

Definition 1 (DT-LPV system): Let $\mathbb{T}=\mathbb{Z}$. The parameter-varying system $\mathcal{S}$ is called LPV, if the following conditions are satisfied:

- $\mathbb{W}$ is a vector-space and $\mathfrak{B}_{p}$ is a linear subspace of $\mathbb{W}^{\mathbb{T}}$ for all $p \in \mathfrak{B}_{\mathbb{P}}$ (linearity).

- For any $(w, p) \in \mathfrak{B}$ and any $\tau \in \mathbb{T}$, it holds that $(w(\cdot+$ $\tau), p(\cdot+\tau)) \in \mathfrak{B}$, in other words $q^{\tau} \mathfrak{B}=\mathfrak{B}$ (timeinvariance).

Note that in terms of Definition 1, for a constant scheduling trajectory, i.e. there exists $\overline{\mathrm{p}} \in \mathbb{P}$ s.t. $p(k)=\overline{\mathrm{p}}$ for all $k \in \mathbb{Z}$, time-invariance of $\mathcal{S}$ implies time-invariance of the corresponding system $\mathcal{G}=\left(\mathbb{T}, \mathbb{W}, \mathfrak{B}_{\overline{\mathrm{p}}}\right)$. Based on this and the linearity condition of $\mathfrak{B}_{p}$, it holds for an LPV system that for each $\overline{\mathrm{p}} \in \mathbb{P}$ the associated system $\mathcal{G}=\left(\mathbb{T}, \mathbb{W}, \mathfrak{B}_{\overline{\mathrm{p}}}\right)$ is an LTI system, which is in accordance with previous definitions of LPV systems [1].

In the sequel, we restrict our attention to DT systems with $\mathbb{W}=\mathbb{R}^{n_{\mathbb{W}}}, n_{\mathbb{W}} \in \mathbb{N}$ and with $\mathbb{P}$ a closed subset of $\mathbb{R}^{n_{\mathbb{P}}}, n_{\mathbb{P}} \in \mathbb{N}$. In fact, we consider LPV systems described by finite order linear difference equations with parametervarying effects in the coefficients. A property of such systems is that the behavior $\mathfrak{B}$ is complete, i.e. $((w, p) \in \mathfrak{B} \Leftrightarrow$ $\left.\left.\left.(w, p)\right|_{\left[k_{0}, k_{1}\right]} \in \mathfrak{B}\right|_{\left[k_{0}, k_{1}\right]}, \forall\left[k_{0}, k_{1}\right] \subset \mathbb{Z}\right)$.

\section{SySTEM REPRESENTATIONS}

\section{A. Algebraic preliminaries}

As a first step we introduce difference equations with varying coefficients as the representation of the behavior $\mathfrak{B}$, in order to develop IO and SS representations. The introduced difference equations will be associated with polynomials defining a ring where equivalence of representations and other concepts of the system theory can be characterized by simple algebraic manipulations. To develop this polynomial ring, we introduce the sets $\mathcal{R}_{n}$ of so called real meromorphic functions $f: \mathbb{R}^{n} \rightarrow \mathbb{R}$ that can be written as the quotient of two analytic (holomorphic) functions [10]. Furthermore we restrict $\mathcal{R}_{n}$ to essential $n$-dimensional functions, i.e. functions that are not equivalent to a function in $\mathcal{R}_{n^{\prime}}$ with $n^{\prime}<n$. We define the collection of these sets by $\mathcal{R}=$ $\cup_{n \in \mathbb{N}} \mathcal{R}_{n}$. It can be shown that $\mathcal{R}$ is a field [11].

The function class $\mathcal{R}$ will be used as the collection of coefficient functions (like $\{A, \ldots, D\}$ and $\left\{a_{i}, b_{j}\right\}$ in (1ab) and (2)) for the representations. This class encompasses a wide range of functions, including polynomials, rational functions etc. These functions are used to enable a distinction between dynamic scheduling dependency of the coefficients and the dynamic relation between the signals of the system. For a given $\mathbb{P}$ with dimension $n_{\mathbb{P}}$, let $r \in \mathcal{R}_{n}$, so we can talk about $r\left(\mathrm{x}_{1}, \cdots, \mathrm{x}_{n}\right)$. Rename the variables $\mathrm{x}_{i}$ to 'new' variables $\zeta_{i j}$ in the following order,

$$
\left\{\zeta_{0,1}, \ldots, \zeta_{0, n_{\mathbb{P}}}, \zeta_{1,1}, \ldots, \zeta_{1, n_{\mathbb{P}}}, \zeta_{-1,1}, \ldots, \zeta_{-1, n_{\mathbb{P}}}, \zeta_{2,1}, \ldots\right\}
$$

and for a given scheduling signal $p$, associate the variable $\zeta_{i j}$ with $q^{i} p_{j}$. For this association we introduce the operator $\diamond:\left(\mathcal{R}, \mathfrak{B}_{\mathbb{P}}\right) \rightarrow \mathbb{R}^{\mathbb{Z}}$, defined by $r \diamond p=r\left(p, q p, q^{-1} p, \ldots\right)$.

The value of a ( $p$-dependent) coefficient in an LPV system representation is now given by an operation $(r \diamond p)(k)$.

Example 2 (Coefficient function): Let $\mathbb{P}=\mathbb{R}^{n_{\mathbb{P}}}$ with $n_{\mathbb{P}}=2$. Consider the real-meromorphic coefficient function $r: \mathbb{R}^{3} \rightarrow \mathbb{R}$, defined as

$$
r\left(\mathrm{x}_{1}, \mathrm{x}_{2}, \mathrm{x}_{3}\right)=\frac{1+\mathrm{x}_{3}}{1-\mathrm{x}_{2}} .
$$

Then for a scheduling signal $p: \mathbb{Z} \rightarrow \mathbb{R}^{2}$ :

$$
(r \diamond p)(k)=r\left(p_{1}, p_{2}, q p_{1}\right)(k)=\frac{1+p_{1}(k+1)}{1-p_{2}(k)} .
$$

In the sequel the (time-varying) coefficient sequence $(r \diamond p)$ will be used to operate on a signal $w$ (like $a_{i}(p)$ in (2)), giving the varying coefficient sequence of the representations. In this respect an important property of the $\diamond$ operation is that multiplication with the shift operator $q$ is not commutative, in 
other words $q(r \diamond p) \neq(r \diamond p) q$. To handle this multiplication, for $r \in \mathcal{R}$ we define the shift operations $\vec{r}, \overleftarrow{r}$ as

$$
\begin{aligned}
& \vec{r}=r^{\prime} \in \mathcal{R} \quad \text { s.t. } \quad r^{\prime} \diamond p=r \diamond(q p), \\
& \overleftarrow{r}=r^{\prime \prime} \in \mathcal{R} \quad \text { s.t. } \quad r^{\prime \prime} \diamond p=r \diamond\left(q^{-1} p\right),
\end{aligned}
$$

for $p \in\left(\mathbb{R}^{n_{\mathrm{P}}}\right)^{\mathbb{Z}}$. With these notions we can write $q r=\vec{r} q$ and $q^{-1} r=\overleftarrow{r} q^{-1}$ which corresponds to

$q(r \diamond p) w=(\vec{r} \diamond p) q w \quad$ and $\quad q^{-1}(r \diamond p) w=(\overleftarrow{r} \diamond p) q^{-1} w$

in the signal level.

The considered operator $\diamond$ can straightforwardly be extended to matrix functions $r \in \mathcal{R}^{n_{r} \times n_{\mathrm{W}}}$ where the operation $\diamond$ is applied to each scalar entry of the matrix. Let $\mathcal{R}[\xi]^{n_{r} \times n_{\mathrm{W}}}$ be the ring of matrix polynomials in the indeterminant $\xi$ and with coefficients in $\mathcal{R}^{n_{\mathrm{r}} \times n_{\mathrm{W}}}$, then a parameter-varying (PV) difference equation with $n_{\mathrm{r}}$ rows and signal dimension $n_{\mathbb{W}}$ is defined as follows:

$$
(R(q) \diamond p) w:=\sum_{i=0}^{n_{\xi}}\left(r_{i} \diamond p\right) q^{i} w=0,
$$

where $R=\sum_{i=0}^{n_{\xi}} r_{i} q^{i}, n_{\xi}=\operatorname{deg}(R)$, and $r_{i} \in \mathcal{R}^{n_{\mathrm{r}} \times n_{\mathrm{W}}}$. In this notation the shift operator $q$ operates on the signal $w$, while the operation $\diamond$ takes care of the time/scheduledependent coefficient sequence. Since the indeterminant $\xi$ is associated with $q$, multiplication with $\xi$ is noncommutative on $\mathcal{R}[\xi]^{n_{\mathrm{r}} \times n_{\mathrm{W}}}$, i.e. $\xi r=\vec{r} \xi$ and $r \xi=\xi \overleftarrow{r}$.

It can be shown that with the above defined noncommutative multiplicative rules $\mathcal{R}[\xi]$ defines an Ore algebra [12] and it is a left and right Euclidian domain [13]. With these algebraic properties, there exists a duality between the solution spaces of PV difference equations and the polynomial modules associated with them, which is implied by a so called injective cogenerator property. This has been shown for the solution spaces of the polynomial ring over $\mathcal{R}_{1}$ in [5]. Due to the fact that all required algebraic properties are satisfied for $\mathcal{R}[\xi]$, the proof of the injective cogenerator property similarly follows in this case. Based on these facts, we will omit the rather heavy technicalities to prove certain theorems in the following discussion as all proofs similarly follows in $\mathcal{R}[\xi]$ as in $\mathcal{R}_{1}[\xi]$.

Due to the fact that $\mathcal{R}[\xi]$ is a right and left Euclidean domain, there exists left and right division by remainder. This means, that if $R_{1}, R_{2} \in \mathcal{R}[\xi]$ with $\operatorname{deg}\left(R_{1}\right) \geq \operatorname{deg}\left(R_{2}\right)$ and $R_{2} \neq 0$, then there exist unique polynomials $R^{\prime}, R^{\prime \prime} \in \mathcal{R}[\xi]$ such that

$$
R_{1}=R_{2} R^{\prime}+R^{\prime \prime} \text {. }
$$

wher $\operatorname{deg}\left(R_{2}\right)>\operatorname{deg}\left(R^{\prime \prime}\right)$.

\section{B. Kernel representation}

Using these concepts, we can introduce the kernel representation (KR) of an LPV system in the form of (6). More precisely, we call (6) a representation of the LPV system $\mathcal{S}=(\mathbb{Z}, \mathbb{P}, \mathbb{W}, \mathfrak{B})$ with scheduling signal $p$ and signals $w$ if

$$
\mathfrak{B}=\left\{(w, p) \in\left(\mathbb{R}^{n_{\mathbb{W}}} \times \mathbb{R}^{n_{\mathbb{P}}}\right)^{\mathbb{Z}} \mid(R(q) \diamond p) w=0\right\} .
$$

We will only consider LPV systems which have a kernel representation and we will show that this system class includes all LPV systems that can be described in the form of (1a-b) and (2) (in terms of manifest behavior).

An important concept to be established, is full row rank KR representations. Denote by $\operatorname{span}_{\mathcal{R}}^{\text {row }}(R)$ and $\operatorname{span}_{\mathcal{R}}^{\text {col }}(R)$ the subspace spanned by the rows (columns) of $R \in \mathcal{R}[\xi]^{\cdot \times}$, viewed as a linear space of polynomial vector functions with coefficients in $\mathcal{R} \cdot \times \cdot$. Then it can be shown that

$$
\operatorname{rank}(R)=\operatorname{dim}\left(\operatorname{span}_{\mathcal{R}}^{\text {row }}(R)\right)=\operatorname{dim}\left(\operatorname{span}_{\mathcal{R}}^{\text {col }}(R)\right) .
$$

Based on the concept of rank, the following theorem holds:

Theorem 1 (Full row rank KR representation): Let $\mathfrak{B}$ be given with a KR representation (6). Then, $\mathfrak{B}$ can also be represented by a $R^{\prime} \in \mathcal{R}[\xi]^{\cdot \times n_{\mathrm{W}}}$ with full row rank.

Due to the algebraic properties of $\mathcal{R}[\xi]$, the proof of this theorem follows along similar lines as in [5]. Like in the LTI case, the concept of minimality for KR representations can be defined based on the full row rank of the associated matrix polynomials.

\section{IO representation}

For practical applications a partitioning of the signals $w$ into input signals $u \in\left(\mathbb{R}^{n_{\Psi}}\right)^{\mathbb{Z}}$ and output signals $y \in\left(\mathbb{R}^{n_{\mathbb{V}}}\right)^{\mathbb{Z}}$, i.e. $w=\operatorname{col}(u, y)$, is often convenient. Such a partitioning is called an IO partition of $\mathcal{S}$ if

1) $u$ is free, i.e. for all $u \in\left(\mathbb{R}^{n_{\mathbb{U}}}\right)^{\mathbb{Z}}$ and $p \in \mathfrak{B}_{\mathbb{P}}$, there exists a $y \in\left(\mathbb{R}^{n_{\mathbb{Y}}}\right)^{\mathbb{Z}}$ such that $(\operatorname{col}(u, y), p) \in \mathfrak{B}$.

2) $y$ does not contain any further free component, i.e. given $u$, none of the components of $y$ can be chosen freely for every $p \in \mathfrak{B}_{\mathbb{P}}$ (maximally free).

Using an IO partition we can define the $I O$ representation of $\mathcal{S}$ as

$$
\left(R_{\mathrm{y}}(q) \diamond p\right) y=\left(R_{\mathrm{u}}(q) \diamond p\right) u,
$$

where $R_{\mathrm{u}}$ and $R_{\mathrm{y}}$ are matrix polynomials with meromorphic coefficients, and where $R_{\mathrm{y}}$ is full row rank and $\operatorname{deg}\left(R_{\mathrm{y}}\right) \geq$ $\operatorname{deg}\left(R_{\mathrm{u}}\right)$. Using the same type of decomposition as in (6), we derive the following form of an IO representation

$$
\sum_{i=0}^{n_{\mathrm{a}}}\left(a_{i} \diamond p\right) q^{i} y=\sum_{j=0}^{n_{\mathrm{b}}}\left(b_{j} \diamond p\right) q^{j} u .
$$

It is apparent that (11) is the 'dynamic-dependent' counterpart of (2).

\section{State-space representation}

In modeling dynamical systems the use of auxiliary variables (often called latent variables) is common. The natural counterpart of (6) to cope with this is

$$
\left(R_{\mathrm{w}}(q) \diamond p\right) w=\left(R_{\mathrm{L}}(q) \diamond p\right) w_{\mathrm{L}},
$$

where $w_{\mathrm{L}}: \mathbb{Z} \rightarrow \mathbb{R}^{n_{\mathrm{L}}}$ are the latent variables and $R_{\mathrm{L}} \in \mathcal{R}[\xi]^{n_{\mathrm{r}} \times n_{\mathrm{L}}}$. The set of equations (12) is called a latent variable representation of the latent variable system $\left(\mathbb{Z}, \mathbb{R}^{n_{\mathbb{P}}}, \mathbb{R}^{n_{\mathrm{W}}} \times \mathbb{R}^{n_{\mathrm{L}}}, \mathfrak{B}_{\mathrm{L}}\right)$, where the full behavior $\mathfrak{B}_{\mathrm{L}}$ is composed of the trajectories of $\left(w, w_{\mathrm{L}}, p\right)$ satisfying (12) and inducing the manifest behavior $\mathfrak{B}=\pi_{(w, p)} \mathfrak{B}_{\mathrm{L}}$. Based 
on the result of [5] for LTV systems, it can be proven that elimination of latent variables is always possible on $\mathcal{R}[\xi]^{\cdot \times}$. This elimination property implies that if (12) corresponds to a latent variable LPV system, then there exists a $R^{\prime} \in$ $\mathcal{R}[\xi]^{\cdot \times n_{W}}$ which defines a LPV-KR representation of $\mathfrak{B}$.

Now it is possible to define the concept of state for LPV systems. Let $\left(\mathbb{Z}, \mathbb{R}^{n_{\mathbb{P}}}, \mathbb{R}^{n_{\mathbb{W}}} \times \mathbb{R}^{n_{\mathrm{L}}}, \mathfrak{B}_{\mathrm{L}}\right)$ be a LPV latent variable system. Then the latent variable $w_{\mathrm{L}}$ is a state if for every $k_{0} \in \mathbb{Z}$ and $\left(w_{1}, w_{\mathrm{L}, 1}, p\right),\left(w_{2}, w_{\mathrm{L}, 2}, p\right) \in \mathfrak{B}_{\mathrm{L}}$ with $w_{\mathrm{L}, 1}\left(k_{0}\right)=w_{\mathrm{L}, 2}\left(k_{0}\right)$ it follows that the concatenation of these signals at $k_{0}$ satisfies

$$
\left(w_{1}, w_{\mathrm{L}, 1}, p\right) \hat{k}_{k_{0}}\left(w_{2}, w_{\mathrm{L}, 2}, p\right) \in \mathfrak{B}_{\mathrm{L}} .
$$

To decide whether a latent variable is a state, the following theorem is important:

Theorem 2 (State-kernel form): The latent variable $w_{\mathrm{L}}$ is a state, iff there exist matrices $r_{\mathrm{w}} \in \mathcal{R}^{n_{\mathrm{r}} \times n_{\mathbb{W}}}$ and $r_{0}, r_{1} \in$ $\mathcal{R}^{n_{\mathrm{r}} \times n_{\mathrm{L}}}$ such that the full behavior $\mathfrak{B}_{\mathrm{L}}$ has the kernel representation:

$$
r_{\mathrm{w}} w+r_{0} w_{\mathrm{L}}+r_{1} q w_{\mathrm{L}}=0 .
$$

The proof of this Theorem follows similarly as in the LTI case (see [11]). Now we can formulate the DT state-space representation, based on an IO partition $(u, y)$, as a firstorder parameter-varying difference equation system in the state variable $x: \mathbb{Z} \rightarrow \mathbb{X}$ as:

$$
\begin{aligned}
q x & =(A \diamond p) x+(B \diamond p) u, \\
y & =(C \diamond p) x+(D \diamond p) u,
\end{aligned}
$$

where $\mathbb{X}=\mathbb{R}^{n_{\mathbb{X}}}$ is called the state space and

$$
\mathfrak{B}_{\mathrm{SS}}=\left\{(u, x, y, p) \in(\mathbb{U} \times \mathbb{X} \times \mathbb{Y} \times \mathbb{P})^{\mathbb{Z}} \mid(15 \mathrm{a}-\mathrm{b}) \text { hold }\right\},
$$

is the full behavior, and

$$
\left[\begin{array}{c|c}
A & B \\
\hline C & D
\end{array}\right] \in\left[\begin{array}{l|l}
\mathcal{R}^{n_{\mathbb{X}} \times n_{\mathbb{X}}} & \mathcal{R}^{n_{\mathbb{X}} \times n_{\mathbb{U}}} \\
\hline \mathcal{R}^{n_{\mathbb{Y}} \times n_{\mathbb{X}}} & \mathcal{R}^{n_{\mathbb{Y}} \times n_{\mathbb{U}}}
\end{array}\right],
$$

represents the meromorphic PV state-space matrices (matrix functions) of the representation. It is apparent that (15a-b) are the 'dynamic-dependent' counterparts of (1a-b).

\section{EQUiVALENCE RELATIONS}

Using the behavioral framework, it is possible to consider equivalence of kernel representations, IO representations and state-space forms via equality of the represented behaviors.

\section{A. Equivalent kernel forms}

In the LTI case, two DT kernel representations are equivalent, i.e. they define the same system, if their associated behavior is equal. Note that in $\mathcal{R}[\xi]$ left multiplication of polynomial $R$ by $r \in \mathcal{R}$ can alter the associated behavior of $R$ in terms of (6) as some scheduling trajectories from the set of solutions might be excluded due to possible singularity of $r$. However, the rest of the behavior remains the same.

To define equality of LPV-KR representations with the previous phenomenon of singularity in mind, define the restriction of $\mathfrak{B}$ to $\overline{\mathfrak{B}}_{\mathbb{P}} \subseteq \mathfrak{B}_{\mathbb{P}}$ as

$$
\left.\mathfrak{B}\right|_{\overline{\mathfrak{B}}_{\mathbb{P}}}=\left\{(w, p) \in \mathfrak{B} \mid p \in \overline{\mathfrak{B}}_{\mathbb{P}}\right\} .
$$

The equivalence of LPV-KR representations can now be introduced in an almost everywhere sense:

Definition 2 (Equivalent KR representations): Two kernel representations with polynomials $R, R^{\prime} \in \mathcal{R}[\xi]^{\bullet \times n_{\mathbb{W}}}$ and behaviors $\mathfrak{B}, \mathfrak{B}^{\prime} \subseteq\left(\mathbb{R}^{n_{\mathbb{W}}} \times \mathbb{R}^{n_{\mathbb{P}}}\right)^{\mathbb{Z}}$ are called equivalent if $\left.\mathfrak{B}\right|_{\mathfrak{B}_{\mathbb{P}} \cap \mathfrak{B}_{\mathbb{P}}^{\prime}}=\left.\mathfrak{B}^{\prime}\right|_{\mathfrak{B}_{\mathbb{P}} \cap \mathfrak{B}_{\mathbb{P}}^{\prime}}$, i.e. their behaviors are equal for all possible mutually valid trajectories of $p$.

To characterize when two KR representations are equivalent we introduce left/right unimodular transformations just like in the LTI case. We call a $M \in \mathcal{R}[\xi]^{n \times n}$ unimodular, if there exists a $M^{\dagger} \in \mathcal{R}[\xi]^{n \times n}$, such that $M^{\dagger}(\xi) M(\xi)=I$ and $M(\xi) M^{\dagger}(\xi)=I$. Based on this concept it is possible to show that the following theorem holds in the LPV case:

Theorem 3 (Unimodular transfor.): Let $R \in \mathcal{R}[\xi]^{n_{\mathrm{r}} \times n_{W}}$ and $M^{\prime} \in \mathcal{R}[\xi]^{n_{\mathrm{r}} \times n_{\mathrm{r}}}, M^{\prime \prime} \in \mathcal{R}[\xi]^{n_{\mathbb{W}} \times n_{\mathbb{W}}}$ with $M^{\prime}, M^{\prime \prime}$ unimodular. For a given $n_{\mathbb{P}} \in \mathbb{N}$, define $R^{\prime}=M^{\prime} R$ and $R^{\prime \prime}=R M^{\prime \prime}$. Denote the behaviors corresponding to $R, R^{\prime}$ and $R^{\prime \prime}$ by $\mathfrak{B}, \mathfrak{B}^{\prime}$ and $\mathfrak{B}^{\prime \prime}$ with scheduling space $\mathbb{P} \subseteq \mathbb{R}^{n_{\mathbb{P}}}$ and signal space $\mathbb{W}=\mathbb{R}^{n_{\mathbb{W}}}$. Then $\left.\mathfrak{B}\right|_{\mathfrak{B}_{\mathbb{P}} \cap \mathfrak{B}_{\mathbb{P}}^{\prime}}=\left.\mathfrak{B}^{\prime}\right|_{\mathfrak{B}_{\mathbb{P}} \cap \mathfrak{B}_{\mathbb{P}}^{\prime}}$ while $\left.\mathfrak{B}\right|_{\mathfrak{B}_{\mathbb{P}} \cap \mathfrak{B}_{\mathbb{P}}^{\prime \prime}}$ and $\left.\mathfrak{B}^{\prime \prime}\right|_{\mathfrak{B}_{\mathbb{P}} \cap \mathfrak{B}_{\mathbb{P}}^{\prime \prime}}$ are isomorphic.

The proof of this theorem similarly follows as in $\mathcal{R}_{1}[\xi]$ (see [5] and [6]).

\section{B. Equivalent IO forms}

The introduced equivalency concept generalizes to LPVIO representations. Let $R_{\mathrm{u}}, R_{\mathrm{u}}^{\prime} \in \mathcal{R}[\xi]^{n_{\mathbb{Y}} \times n_{\mathbb{U}}}$ and $R_{\mathrm{y}}, R_{\mathrm{y}}^{\prime} \in$ $\mathcal{R}[\xi]^{n_{\mathbb{Y}} \times n_{\mathbb{Y}}}$ with $R_{\mathrm{y}}, R_{\mathrm{y}}^{\prime}$ full row rank, $\operatorname{deg}\left(R_{\mathrm{y}}\right) \geq \operatorname{deg}\left(R_{\mathrm{u}}\right)$, and $\operatorname{deg}\left(R_{\mathrm{y}}^{\prime}\right) \geq \operatorname{deg}\left(R_{\mathrm{u}}^{\prime}\right)$. For a given $n_{\mathbb{P}} \in \mathbb{N}$, we call the LPV-IO representations defined via $\left(R_{\mathrm{y}}, R_{\mathrm{u}}\right)$ and $\left(R_{\mathrm{y}}^{\prime}, R_{\mathrm{u}}^{\prime}\right)$ equivalent if there exists a unimodular matrix $M \in \mathcal{R}[\xi]^{n_{\mathbb{Y}} \times n_{\mathbb{Y}}}$ such that

$$
R_{\mathrm{y}}^{\prime}=M R_{\mathrm{y}} \quad \text { and } \quad R_{\mathrm{u}}^{\prime}=M R_{\mathrm{u}} .
$$

\section{Equivalent state-space forms}

We can also generalize the equivalency concept to LPVSS representations. To do so, we first have to clarify statetransformations in the LPV case.

By definition, the full behavior of LPV-SS representation is represented by a zero-order and a first-order polynomial matrix $R_{\mathrm{w}} \in \mathcal{R}[\xi]^{n_{\mathrm{r}} \times\left(n_{\mathbb{Y}}+n_{\mathbb{U}}\right)}$ and $R_{\mathrm{L}} \in \mathcal{R}[\xi]^{n_{\mathrm{r}} \times n_{\mathbb{X}}}$ in the form of

$$
\left(R_{\mathrm{w}}(q) \diamond p\right) \operatorname{col}(u, y)=\left(R_{\mathrm{L}}(q) \diamond p\right) x .
$$

Similar to the LTI case, left and right side multiplication of $R_{\mathrm{w}}$ and $R_{\mathrm{L}}$ with unimodular $M_{1} \in \mathcal{R}[\xi]^{n_{\mathrm{r}} \times n_{\mathrm{r}}}$ and $M_{2} \in$ $\mathcal{R}[\xi]^{n_{\mathbb{X}} \times n_{\mathbb{X}}}$ leads to

$$
R_{\mathrm{w}}^{\prime}=M_{1} R_{\mathrm{w}}, \quad R_{\mathrm{L}}^{\prime}=M_{1} R_{\mathrm{L}} M_{2} .
$$

In terms of Theorem 3, the resulting polynomials $R_{\mathrm{w}}^{\prime}$ and $R_{\mathrm{L}}^{\prime}$ define an equivalent latent variable representation of $\mathcal{S}$, where the new latent variable is given as

$$
x^{\prime}=\left(M_{2}^{\dagger}(q) \diamond p\right) x .
$$

To guarantee that the resulting latent variable representation qualifies as a SS representation, $R_{\mathrm{L}}^{\prime}$ needs to be monic and $\operatorname{deg}\left(R_{\mathrm{w}}^{\prime}\right)=0$ with $\operatorname{deg}\left(R_{\mathrm{L}}^{\prime}\right)=1$ must be satisfied. This 
implies that the unimodular matrices must have zero order, i.e. $M_{1} \in \mathcal{R}^{n_{\mathrm{r}} \times n_{\mathrm{r}}}$ and $M_{2} \in \mathcal{R}^{n_{\mathbb{X}} \times n_{\mathbb{X}}}$, and $M_{1}$ must have a special structure in order to guarantee that $R^{\prime}$ and $R_{\mathrm{L}}^{\prime}$ correspond to an equivalent SS representation. In that case, (20) is called a state-transformation and $T=M_{2}^{\dagger}$ is called the state transformation matrix resulting in

$$
x^{\prime}=(T \diamond p) x .
$$

A major difference with respect to LTI state-transformations is that, in the LPV case, $T$ is inherently dependent on $p$ and this dependence is dynamic, i.e. $T \in \mathcal{R}^{n_{\mathbb{X}} \times n_{\mathbb{X}}}$. Additionally it can be shown that an invertible $T \in \mathcal{R}^{n_{\mathbb{X}} \times n_{\mathbb{X}}}$ used as a statetransformation is always equivalent with a right and leftside multiplication by unimodular matrix functions yielding a valid SS representation of the LPV system. Based on this we call two SS representations equivalent if their states can be related via an invertible state-transformation (21).

Consider an LPV-SS representation given by (15a-b). Let $T \in \mathcal{R}^{n_{\mathbb{X}} \times n_{\mathbb{X}}}$ be an invertible matrix function and consider $x^{\prime}$, given by (21), as a new state variable. It is immediate that substitution of (21) into (15a) gives

$$
q\left(T^{-1} \diamond p\right) x^{\prime}=(A \diamond p)\left(T^{-1} \diamond p\right) x^{\prime}+(B \diamond p) u .
$$

This yields that the equivalent LPV-SS representation reads as

$$
\left[\begin{array}{c|c}
\vec{T} A T^{-1} & \vec{T} B \\
\hline C T^{-1} & D
\end{array}\right] .
$$

Based on the state-transformations developed and the concept of state-observability and reachability matrices, the classical canonical forms can also be defined (see [4], [11]).

\section{EQUIVALENCE TRANSFORMATIONS}

Next, we establish the concept of equivalence among statespace and IO representations.

\section{A. State-space to IO}

As a consequence of the elimination property on $\mathcal{R}[\xi]^{\cdot \times}$, for any latent variable representation (18), there exists a unimodular matrix $M \in \mathcal{R}[\xi]^{n_{\mathrm{r}} \times n_{\mathrm{r}}}$ such that

$$
M R_{\mathrm{w}}=\left[\begin{array}{c}
R_{\mathrm{w}}^{\prime} \\
R_{\mathrm{w}}^{\prime \prime}
\end{array}\right], \quad M R_{\mathrm{L}}=\left[\begin{array}{c}
R_{\mathrm{L}}^{\prime} \\
0
\end{array}\right],
$$

with $R_{\mathrm{L}}^{\prime}$ of full row rank. Then the behavior defined by $\left(R_{\mathrm{w}}^{\prime \prime}(\xi) \diamond p\right) w=0$ is equal to the manifest behavior of (18). We can use this result to establish an IO realization of a given SS representation (15a-b) by writing it in the latent form

$$
R_{\mathrm{w}}(q)=\left[\begin{array}{cc}
0 & B \\
-I & D
\end{array}\right], \quad R_{\mathrm{L}}(q)=\left[\begin{array}{c}
I q-A \\
-C
\end{array}\right],
$$

and formulate the unimodular transformation

$$
M(\xi)=\left[\begin{array}{ll}
M_{11}(\xi) & M_{12}(\xi) \\
M_{21}(\xi) & M_{22}(\xi)
\end{array}\right]
$$

such that

$$
M_{21}(\xi)(I \xi-A)-M_{22}(\xi) C=0 .
$$

This yields that

$$
\underbrace{\left[\begin{array}{cc}
* & * \\
-M_{21}(\xi) & M_{21}(\xi) B+M_{22}(\xi) D
\end{array}\right]}_{M(\xi) R_{\mathrm{w}}(\xi)}=\underbrace{\left[\begin{array}{c}
* \\
0
\end{array}\right]}_{M(\xi) R_{\mathrm{L}}(\xi)},
$$

and the resulting $R^{\prime}=\left[\begin{array}{ll}-M_{21} & M_{21} B+M_{22} D\end{array}\right]$ is in the form of an output side polynomial $R_{\mathrm{y}}=M_{21}$ and an input side polynomial $R_{\mathrm{u}}=M_{21} B+M_{22} D$.

Consider a LPV-SS representation with state dimension $n_{\mathbb{X}}$. Due to the elimination property, it holds that there exists a unique monic polynomial $\bar{R}_{\mathrm{y}} \in \mathcal{R}[\xi]^{n_{\mathbb{Y}} \times n_{\mathbb{Y}}}$ with $\operatorname{deg}\left(\bar{R}_{\mathrm{y}}\right)=n_{\mathbb{X}}$ and a unique $\bar{R}_{\mathrm{u}} \in \mathcal{R}[\xi]^{n_{\mathbb{Y}} \times n_{\mathbb{X}}}$ with $\operatorname{deg}\left(\bar{R}_{\mathrm{u}}\right) \leq n_{\mathbb{X}}-1$ such that

$$
\bar{R}_{\mathrm{y}}(\xi) C=\bar{R}_{\mathrm{u}}(\xi)(I \xi-A)
$$

Let $R_{\mathrm{c}}=\operatorname{diag}\left(r_{1}, \ldots, r_{n_{\mathbb{Y}}}\right), r_{i} \in \mathcal{R}[\xi]$, be the greatest common divisor of $\bar{R}_{\mathrm{y}}$ and $\bar{R}_{\mathrm{u}} B$ such that there exist $R_{\mathrm{y}}, R_{\mathrm{u}} \in \mathcal{R}[\xi]$ satisfying

$$
\begin{aligned}
& R_{\mathrm{c}}(\xi) R_{\mathrm{y}}(\xi)=\bar{R}_{\mathrm{y}}(\xi) \\
& R_{\mathrm{c}}(\xi) R_{\mathrm{u}}(\xi)=\bar{R}_{\mathrm{u}}(\xi) B+\bar{R}_{\mathrm{y}}(\xi) D .
\end{aligned}
$$

Then the IO representation, given by

$$
\left(R_{\mathrm{y}}(q) \diamond p\right) y=\left(R_{\mathrm{u}}(q) \diamond p\right) u
$$

defines a behavior which is equal to the manifest behavior of (15a-b).

Note that the algorithm defined by (26) and (27a-b) is structurally similar to the LTI case, but it is more complicated as it involves multiplication with the time operators on the coefficients. Thus, this transformation can result in an increased complexity (like dynamic dependence) of the coefficient functions in the equivalent IO representation.

\section{B. IO to state-space}

Finding an equivalent SS representation of a given IO representation follows by constructing a state mapping. This construction can be seen as the reverse operation of the latent variable elimination. The aim is to introduce a latent variable into (28) such that it satisfies the state property, i.e. it defines a SS representation (Theorem 2). Similar to the LTI case, the central idea of such a state construction is the cut-andshift-map $\varrho_{-}: \mathcal{R}[\xi]^{\cdot \times \cdot} \rightarrow \mathcal{R}[\xi]^{\cdot \times \cdot}$ that acts on polynomial matrices as:

$$
\varrho_{-}(\underbrace{r_{0}+r_{1} \xi+\ldots+r_{n} \xi^{n}}_{R(\xi)})=\overleftarrow{r_{1}}+\ldots+\overleftarrow{r_{n}} \xi^{n-1}
$$

This operator can be seen as an intuitive way to introduce state variables for a kernel representation associated with $R$, as $w_{\mathrm{L}}=\varrho_{-}(R(q) \diamond p) w$ implies that

$$
(R(q) \diamond p) w=\left(r_{0} \diamond p\right) w+q w_{\mathrm{L}} .
$$


Repeated use of $\varrho_{-}$and stacking the resulting polynomial matrices gives

$$
\underbrace{\left[\begin{array}{c}
\varrho_{-}(R) \\
\varrho_{-}^{2}(R) \\
\vdots \\
\varrho_{-}^{n-2}(R) \\
\varrho_{-}^{n-1}(R)
\end{array}\right]}_{\Sigma_{-}(R)}(\xi)=\left[\begin{array}{c}
r_{1}^{[1]}+\ldots+r_{n-1}^{[1]} \xi^{n-2}+\xi^{n-1} \\
r_{2}^{[2]}+\ldots+r_{n-1}^{[2]} \xi^{n-3}+\xi^{n-2} \\
\vdots \\
r_{n-1}^{[n-1]}+\xi \\
1
\end{array}\right]
$$

where $r_{i}^{[j]}$ denotes the backward shift operation applied on $r_{i}$ for $j$-times. In case $R \in \mathcal{R}[\xi]^{n_{\mathrm{r}} \times n_{\mathbb{W}}}$ with $n_{\mathrm{r}}=1$, the rows of $\Sigma_{-}$are independent, thus it can be shown that $X=\Sigma_{-}(R)$ defines a minimal state-map in the form of

$$
x=(X(q) \diamond p) w .
$$

In other cases (MIMO case), independent rows of $\Sigma_{-}(R)$ are selected to formulate $X$, but this selection is generally not unique. Later it is shown that a given state-map implies a unique SS representation. Before that, we characterize all possible minimal state maps that lead to an equivalent SS representation.

Denote the left-side multiplication of $R(\xi)$ by $\xi$ as $\varrho_{+}$and introduce module $_{\mathcal{R}[\xi]}(R)$ as the left module in $\mathcal{R}[\xi]^{n_{\mathrm{r}} \times n_{\mathbb{W}}}$ spanned by the rows of $R \in \mathcal{R}[\xi]^{n_{\mathrm{r}} \times n_{\mathbb{W}}}$ :

$$
\operatorname{module}_{\mathcal{R}[\xi]}(R)=\operatorname{span}_{\mathcal{R}}^{\text {row }}\left(\left[\begin{array}{c}
R \\
\varrho_{+}(R) \\
\vdots
\end{array}\right]\right) .
$$

This module represents the set of equivalence classes on $\operatorname{span}_{\mathcal{R}}^{\text {row }}\left(\Sigma_{-}(R)\right)$. Let $X \in \mathcal{R}[\xi]^{\cdot \times n_{\mathbb{W}}}$ be a polynomial matrix with independent rows (full row-rank) and such that

$$
\begin{aligned}
\operatorname{span}_{\mathcal{R}}^{\text {row }}(X) \oplus \text { module }_{\mathcal{R}[\xi]}(R)= \\
\operatorname{span}_{\mathcal{R}}^{\text {row }}\left(\Sigma_{-}(R)\right)+\text { module }_{\mathcal{R}[\xi]}(R) .
\end{aligned}
$$

Then, similar to the LTI case, it is possible to show that $X$ is a minimal state-map of the LPV system $\mathcal{S}$ and it defines a state variable by (30). This way, it is possible to obtain all minimal, equivalent $\mathrm{SS}$ realizations of $\mathcal{S}$ which have a kernel representation associated with $R$.

The next step is to characterize these SS representations with respect to an IO partition. For a given kernel representation associated with the polynomial $R \in \mathcal{R}[\xi]^{n_{\mathrm{r}} \times n_{\mathbb{W}}}$, the input-output partition is characterized by choosing a selector matrix $S_{\mathrm{u}} \in \mathbb{R}^{\cdot \times n_{\mathbb{W}}}$ giving $u=S_{\mathrm{u}} w$ and a complementary matrix $S_{\mathrm{y}} \in \mathbb{R}^{\cdot \times n_{\mathbb{W}}}$ giving $y=S_{\mathrm{y}} w$.

Assume that a full row rank $X \in \mathcal{R}[\xi]^{\cdot \times n_{\mathbb{W}}}$ is given which satisfies (32). Then $X$ and $S_{\mathrm{u}}$ jointly lead to

$$
\begin{aligned}
\operatorname{span}_{\mathcal{R}}^{\text {row }}\left(\varrho_{+}(X)\right) \subseteq & \operatorname{span}_{\mathcal{R}}^{\text {row }}(X) \oplus \\
& \operatorname{span}_{\mathcal{R}}^{\text {row }}\left(S_{\mathrm{u}}\right) \oplus \operatorname{module}_{\mathcal{R}[\xi]}(R) .
\end{aligned}
$$

On the other hand, $S_{\mathrm{y}}$ gives

$$
\begin{aligned}
\operatorname{span}_{\mathcal{R}}^{\text {row }}\left(S_{\text {y }}\right) \subseteq \operatorname{span}_{\mathcal{R}}^{\text {row }}(X) \oplus \\
\quad \operatorname{span}_{\mathcal{R}}^{\text {row }}\left(S_{\mathrm{u}}\right) \oplus \text { module }_{\mathcal{R}[\xi]}(R) .
\end{aligned}
$$

These inclusions imply that there exist unique matrix functions $\{A, B, C, D\}$ in $\mathcal{R}^{\cdot \times \cdot}$ and polynomial matrix functions $X_{\mathrm{u}}, X_{\mathrm{y}} \in \mathcal{R}[\xi]^{\cdot \times \cdot}$ with appropriate dimensions such that

$$
\begin{aligned}
\xi X(\xi) & =A X(\xi)+B S_{\mathrm{u}}+X_{\mathrm{u}}(\xi) R(\xi) \\
S_{\mathrm{y}} & =C X(\xi)+D S_{\mathrm{u}}+X_{\mathrm{y}}(\xi) R(\xi) .
\end{aligned}
$$

Then

$$
\left[\begin{array}{l|l}
A & B \\
\hline C & D
\end{array}\right] \in\left[\begin{array}{l|l}
\mathcal{R}^{n_{\mathbb{X}} \times n_{\mathbb{X}}} & \mathcal{R}^{n_{\mathbb{X}} \times n_{\mathbb{U}}} \\
\hline \mathcal{R}^{n_{\mathbb{Y}} \times n_{\mathbb{X}}} & \mathcal{R}^{n_{\mathbb{Y}} \times n_{\mathbb{U}}}
\end{array}\right],
$$

is a minimal state-representation of the LPV system $\mathcal{S}$. This algorithm provides an SS realization of both LPV-IO and LPV-KR representations. Specific choices of $X$ leads to specific canonical forms. Note that a similar algorithm can be deduced for a realization in a image type of representation, i.e. latent variable representation (18) where $R_{\mathrm{w}}(q)=I$.

\section{CONCLUSION}

In this paper, we have extended the behavioral approach to LPV systems in order to lay the foundations of a LPV system theory which provides a clear understanding of this system class and the relations of its representations. We have defined LPV systems as the collection of signal and scheduling trajectories and it has been shown that representations of these systems need dynamic dependence on the scheduling variable. By the use of such system descriptions, it has been proven that equivalence relations and transformations between these descriptions can be developed, giving a common ground where model structures of LPV system identification and concepts of LPV control can be compared, analyzed, and further developed.

\section{REFERENCES}

[1] W. Rugh and J. Shamma, "Research on gain scheduling," Automatica, vol. 36, no. 10, pp. 1401-1425, 2000.

[2] C. W. Scherer, "Mixed $\mathcal{H}_{2} / \mathcal{H}_{\infty}$ control for time-varying and linear parametrically-varying systems," Int. Journal of Robust and Nonlinear Control, vol. 6, no. 9-10, pp. 929-952, 1996.

[3] K. Zhou and J. C. Doyle, Essentials of Robust Control. Prentice-Hall, 1998.

[4] R. Tóth, F. Felici, P. S. C. Heuberger, and P. M. J. Van den Hof, "Discrete time LPV I/O and state space representations, differences of behavior and pitfalls of interpolation," in Proc. of the European Control Conf., Kos, Greece, July 2007, pp. 5418-5425.

[5] E. Zerz, "An algebraic analysis approach to linear time-varying systems," IMA Journal of Mathematical Control and Information, vol. 23, pp. 113-126, 2006.

[6] A. Ilchmann and V. Mehrmann, "A behavioral approach to timevarying linear systems. Part 1: General theory," SIAM Journal of Control Optimization, vol. 44, no. 5, pp. 1725-1747, 2005.

[7] J. C. Willems, "The behavioral approach to open and interconnected systems," IEEE Control Systems Magazine, vol. 27, no. 6, pp. 2-56, 2007.

[8] - "Paradigms and puzzels in the theory of dynamical systems," IEEE Tran. on Automatic Control, vol. 36, no. 3, pp. 259-294, 1991.

[9] J. F. Pommaret, Partial Differential Control Theory, vol. I, Mathematical tools. Kluwer Academic Publishers, 2001.

[10] S. G. Krantz, Handbook of Complex Variables. Birkhäuser Verlag, 1999.

[11] R. Tóth, "Modeling and identification of linear parameter-varying systems, an orthonormal basis function approach," Ph.D. dissertation, Delft University of Technology, 2008.

[12] F. Chyzak and B. Salvy, "Non-commutative elimination in Ore algebras proves multivariate identities," Journal of Symbolic Computation, vol. 26, pp. 187-227, 1998.

[13] P. M. Chon, Free Rings and their relations. Academic Press, 1971. 